\title{
Assessment of Hmis and Pcts Related To Women Deliveires in India: A Case Study of Rajasthan State
}

\author{
Dr. Rama Kant Sharma \\ Research Assistant, Population Research Centre, Near Law College, Mohanlal Sukhadia University Campus, \\ Udaipur-313001.
}

\begin{abstract}
Management of information system helps in strengthening of service deliveries in the health sector as it provides the data which is very important at micro-macro planning and for proper programme implementation. The HMIS (Health Management Information System) and PCTS (Pregnancy, Child Tracking System) both web portals are such system programmes which are developed by the Ministry of Health and Family Welfare, GOI and Department of Medical, health and Family Welfare, GOI (Govt. of Rajasthan) respectively. These portals are not only helpful in proper and timely monitoring of input-output of different interventions but also improve the services for beneficiaries and service providers. These web portals are providing to great extent monthly facilities based of service delivery information. In view of this the present paper aims at assessment of the correctness of reported performance of deliveries and field verification by key informants under HMIS and PCTS programme. Paper is based on primary and secondary data sources.
\end{abstract}

\section{Introduction}

The management of information system helps in strengthening of service deliveries in the health sector. The Government of India (GOI) and Rajasthan Government (RG) both have recognized the importance of disaggregated data on the different interventions of the NRHM. It has also been realized that the data is very important in micro-macro planning and proper program implementation. The HMIS (Health Management Information System) and PCTS (Pregnancy, Child Tracking System) both web portals developed by the Ministry of Health and Family Welfare, GOI and Department of Medical, health and Family Welfare, RG respectively.

These portals are not only helpful in proper and timely monitoring of input-output of different interventions but also improve the services for beneficiaries and service providers. These web portals are providing to great extent monthly facilities based of service delivery information.

Since 2008, facilities wise data uploading has started and improved on HMIS to large extent in the country. Every month more than 250 indicators data are uploading regularly. One of the aims of the NRHM (National Rural Health Mission) is to reduce MMR (Maternal Mortality Ratio) and IMR (Infant Mortality Rate) through better universal access of all round public health services and strengthen public health management with adequate and proper service deliveries at different points. Different data banks, researches, surveys data and portals related to the NRHM implementation at different levels have shown some gradual improvement in several aspects. A sudden change has observed in the institutional deliveries which according to DLHS( District Level Household Survey) III was about 45 percent in 2007-08 which reached at 86 percent in HMIS( Health Management Information System) and PCTS(Pregnancy, Child Tracking System) 2009-10.This result motivated to take up an assessment of the correctness of reported performance of deliveries and field verification by key informants. During discussion with state and districts functionaries it has been explored that as per demand of communities and availability of different public and private facilities have been approved for normal delivery other health and family planning services by state and district authorities under Public-Private Partnership.

Majority of districts and sub-district hospitals, CHCs, PHCs have been providing delivery services for 24 hours in Rajasthan. Some of the selected Sub-Centres have been also approved for normal deliveries in some districts in public health sector.

Private health institutions and clinics for different type of health services; such as delivery, laboratory investigations and FW have also been approved are they are actively involved in service delivery at state and district levels.

\section{Objectives}

1. Find out the causes of up word and down word institutional deliveries.

2. Analyze the role of ANM and ASHA regarding promotion of institutional deliveries in rural areas. 
3. Assess performance of the private and public institutions.

4. Identify lacunae in reporting process of institutional deliveries.

\section{Methodology:}

On first step, before field verification the PCTS and HMIS data of Rajasthan were reviewed and analyzed in the light of objectives. Different records (ANC, Delivery, PNC, Immunization related registers, cards, daily diary and data portals reporting) of five years (2008-13) were reviewed and tried to analyze the deliveries performance.

On second step, 100 key informants including delivered mothers, district \& block medical officers, staff of district and block programme management units, ANM, LHV, and ASHA were interviewed. On the basis of their responses, some causes of over and under reporting, number of up word and down word institutional deliveries and the role of services providers in promotion of institutional deliveries have been analyzed.

\section{Performance by Place of Deliveries: Rajasthan}

Home deliveries were very common in Rajasthan which have changed after implementation of the NRHM. Table-1 reveals that during last five years home deliveries decreased gradually and institutional deliveries increased in each year in Rajasthan. Approximately 2 to 3 percent change of home and institutional deliveries observed in the state.

Table-1: Deliveries proportion by years and place of deliveries in Rajasthan (2008-13).

\begin{tabular}{lccc}
\hline Period & $\begin{array}{c}\text { Home } \\
\%\end{array}$ & $\begin{array}{c}\text { Place of deliveries } \\
\text { Institution } \\
\%\end{array}$ & Total \\
\hline $\mathbf{2 0 0 8 - 0 9}$ & 24.34 & 75.66 & 100.00 \\
$\mathbf{2 0 0 9 - 1 0}$ & 13.75 & 86.25 & 100.00 \\
$\mathbf{2 0 1 0 - 1 1}$ & 11.53 & 88.47 & 100.00 \\
$\mathbf{2 0 1 1 - 1 2}$ & 9.37 & 90.63 & 100.00 \\
$\mathbf{2 0 1 2 - 1 3}$ & 7.37 & 92.3 & 100.00 \\
\hline
\end{tabular}

\section{Performance of safe deliveries: Rajasthan}

On one hand all deliveries conducted at institutions (Public and private) are safe. On the other hand, home deliveries are safe if delivery conducted by trained service providers. Table -2 shows that after implementation of the NRHM, safe deliveries increased by about 11 percent during last five years (2008-2013). In other words, safe deliveries have been upward and unsafe deliveries have been down ward turned. This may be due to door to door approach of service providers, incentive on institutional deliveries and to improvement in reporting system.

Table-2: Performance of safe deliveries in Rajasthan

\begin{tabular}{lrrr}
\hline Years & Safe & Not safe & Total \\
\hline $2008-09$ & $\mathbf{8 4 . 4 3}$ & 15.57 & 100.00 \\
$2009-10$ & 93.87 & 6.13 & 100.00 \\
$2010-11$ & 93.66 & 6.34 & 100.00 \\
$2011-12$ & 94.29 & 5.71 & 100.00 \\
$2012-13$ & 95.40 & 4.62 & 100.00
\end{tabular}

\section{Performance of deliveries by public and private institutions: Rajasthan}

Table-3 reveals that most of the deliveries were conducted at public health institutions during last five years. Overall deliveries performance of public institutions seems good as compared to private. Table also pin pointes a gradual declined of deliveries in public institutions. But, during 2011-12 deliveries at public institutions decreased by two percent and increased by same proportion at private institutions in Rajasthan to total institutional deliveries. 


\section{Performance of SBA and Non SBA home deliveries: Rajasthan}

In Rajasthan home deliveries attended by SBA have gradually decreased and non SBA increased during last three years in Rajasthan.

Table- 4 : Performance of SBA and Non SBA home deliveries in Rajasthan

\begin{tabular}{lccc}
\cline { 2 - 4 } Period & SBA & $\begin{array}{c}\text { Deliveries Attendants } \\
\text { Non-SBA }\end{array}$ & Total \\
\hline $2008-09$ & 36.02 & 63.98 & 100.00 \\
$2009-10$ & 55.41 & 44.59 & 100.00 \\
$2010-11$ & 45.00 & 55.00 & 100.00 \\
$2011-12$ & 39.04 & 60.96 & 100.00 \\
$2012-13$ & 40.60 & 59.40 & 100.00 \\
& & & \\
\hline
\end{tabular}

Table- 4 shows 44.59 percent home deliveries conducted by non safe birth attendants which reached 60.85 percent in 2011-12.During last five years the lowest SBA and the highest non SBA home deliveries were reported in the year 2008-09.

\section{Proportion of C- section deliveries: Rajasthan}

Data of last five years indicate that the proportion of C-section delivery has increased in public and private institutions in the state.

Table -5: Proportion of C-section deliveries by type of health facilities: Rajasthan

\begin{tabular}{lccr}
\hline Period & Public facility & Private facility & Total \\
\hline $2008-09$ & 4.12 & 1.20 & 5.32 \\
$2009-10$ & 3.97 & 4.91 & 8.88 \\
$2010-11$ & 3.67 & 9.39 & 13.06 \\
$2011-12$ & 4.46 & 9.03 & 13.49 \\
$2012-13$ & 5.2 & 13.4 & 18.60 \\
\hline
\end{tabular}

Table -5 reveals that out of the total deliveries only 5.32 percent deliveries were $\mathrm{C}$ - section in the state during 2008-09 which reached up to 18.60 percent during 2012-13. C section deliveries are more in private facilities as compared to public health facilities. It has been observed that better facilities for C-section are available at district hospital and private hospitals at most of the district headquarters in Rajasthan.

\section{Results}

Effective, proper and time bound implementation of the NRHM has been major contributor of service quality and deliveries performance in Rajasthan during last five years. After 2008-09 all round efforts in the light of quantity and quality of human resources-infrastructure development, public- private coordination and cooperation, free test and diagnostic, medicine, diet, monetary incentives and transportation are some of the causes which have directly and indirectly contributed to delivery performance in the state. The government of Rajasthan has been regularly strengthening role and numbers of staff in different cadres of service providers and community link persons through different training on one hand. On the other hand, monetary incentives for different health services were also introduced. Both the cadres of staff have established and developed good repo and relation with the community. This has resulted as improvement in different health indicators. Under Public-Private Partnership (PPP), Government of Rajasthan has not only mobilized and sensitized private sectors to participate in health sector in various manner and different levels their active participation has boosted up. Regularly and day to day monitoring of data portals and field visits in relation to programme implementation plan have been contributing the performance of health indicators. Over all data quality and quantity was not so good and error were more before development of the HMIS and PCTS portals. Development of these softwares and their continuing up gradation will minimize data errors and improved effective service indicators monitoring in the state. 


\section{References:}

[1]. Website: http:/nrhm-mis.nic.in

[2]. Website: http:/ pctsrajmedica. raj.nic.in

[3]. Nagda, B.L. \& Sharma, RamaKant (2011) Level of Reporting of Maternal and Child Health Services Mainly Institutional Delivery and Immunization in the HMIS in Public and Private Institutions, PRC, Udaipur. 